\title{
Monochromatic dark neutrinos and boosted dark matter in noble liquid direct detection
}

\author{
David McKeen and Nirmal Raj \\ TRIUMF, 4004 Wesbrook Mall, Vancouver, British Columbia V6T 2A3, Canada
}

(Received 20 December 2018; published 13 May 2019)

\begin{abstract}
If dark matter self-annihilates into neutrinos or a second component of ("boosted") dark matter that is nucleophilic, the annihilation products may be detected with high rates via coherent nuclear scattering. A future multi-ten-tonne liquid xenon detector such as DARWIN, and a multi-hundred-tonne liquid argon detector, ARGO, would be sensitive to the flux of these particles in complementary ranges of 10-1000 MeV dark matter masses. We derive these sensitivities after accounting for atmospheric and diffuse supernova neutrino backgrounds, and realistic nuclear recoil acceptances. We find that their constraints on the dark neutrino flux may surpass neutrino detectors such as Super-Kamiokande, and that they would extensively probe parametric regions that explain the missing satellites problem in neutrino portal models. The XENON1T and BOREXINO experiments currently restrict the effective baryonic coupling of thermal boosted dark matter to $\lesssim 10-100 \times$ the weak interaction, but DARWIN and ARGO would probe down to couplings 10 times smaller. Detection of boosted dark matter with baryonic couplings $\sim 10^{-3}-10^{-2} \times$ the weak coupling could indicate that the dark matter density profile in the centers of galactic halos become cored, rather than cuspy, through annihilations. This work demonstrates that, alongside liquid xenon, liquid argon direct detection technology would emerge a major player in dark matter searches within and beyond the WIMP paradigm.
\end{abstract}

DOI: $10.1103 /$ PhysRevD.99.103003

\section{INTRODUCTION}

The hunt for the identity of dark matter is a most riveting endeavor. Particle dark matter may reveal itself in products of its self-annihilations, in target recoils in scattering experiments, or as missing momenta in colliders. Annihilation signals hold particular interest because they may contain information about the primordial thermal history of dark matter, and in particular, how much of its measured abundance it owes to freezing out of equilibrium in the early Universe. Conservative upper bounds on the total annihilation cross section of dark matter may be placed by constraining the flux of neutrinos, since they are the least detectable Standard Model (SM) states [1,2].

The future of dark matter searches will depend crucially on direct detection with a panoply of noble liquid detectors, proposed with a view of achieving exposures of $\mathcal{O}(10)$ $\mathcal{O}(1000)$ tonne-years. These are the xenon-based XENONnT [3], LUX-ZEPLIN [4] and the multi-10-ton DARWIN [5], the argon-based DARKSIDE-20K [6], and a multi-100-ton liquid argon detector recently christened "ARGO" [7]. DARWIN and

Published by the American Physical Society under the terms of the Creative Commons Attribution 4.0 International license. Further distribution of this work must maintain attribution to the author(s) and the published article's title, journal citation, and DOI. Funded by SCOAP ${ }^{3}$.
ARGO are billed to be "ultimate detectors" in the direct search for dark matter. By virtue of their large exposures, they are expected to set the best limits on the dark matternucleon scattering cross section, probing all the way down to the high-energy "neutrino floor", i.e., the irreducible background of neutrino fluxes from the atmospheric scattering of cosmic rays and from relic supernovae. By virtue of the large dark matter fluxes they would admit, they would also set the best limits on the dark matter mass, probing up to and beyond Planck masses [8]. In this work, we show that these experiments are also poised to become a leading probe of the neutrino flux from dark matter annihilations. We shall henceforth call neutrinos produced in this way "dark neutrinos".

As we will discuss below, the annihilation channel $\chi \bar{\chi} \rightarrow$ $\nu \bar{\nu}$ has been constrained using data from large-volume neutrino detectors [2,9]; we show that DARWIN and ARGO sensitivities would compete with and better them in the $\sim 10-1000 \mathrm{MeV}$ dark matter mass range. This is not entirely surprising; while neutrino detectors admit larger fluxes and exposures by construction, noble liquid direct detection experiments enjoy enhanced rates thanks to coherent scattering with large nuclei. Moreover, search channels at neutrino detectors are typically sensitive to only $\nu_{e}$ and/or $\bar{\nu}_{e}$ flavors, which may make up but a fraction of the dark neutrino flux, whereas the coherent nuclear scattering channel at direct detection is equally sensitive 
to the flavors $\nu_{e}, \bar{\nu}_{e}, \nu_{\mu}, \bar{\nu}_{\mu}, \nu_{\tau}, \bar{\nu}_{\tau}$. These features have been exploited to determine direct detection sensitivities to signals of neutrinos from a future core-collapse supernova burst [10-13], solar neutrinos [14-17], geoneutrinos [18], and products of dark matter decay [19] and annihilations [20]. Reference [20] used a LUX dataset with 0.027 tonneyears of exposure to constrain dark neutrinos, but we find that these constraints were weaker than those derived from neutrino experiments in [2,9]; the $100-1000$ tonneyear tonne-year data sets at DARWIN and ARGO would reverse this hierarchy of bounds.

Amusingly, a dark neutrino flux could produce a new neutrino floor emerging ahead of the discovery of the standard neutrino floor. Yet, direct detection may not be able to untangle dark neutrino signals from other exotic sources of neutrinos, such as decaying dark matter [19]. Moreover, annual modulation signals are absent. Therefore the true discovery of dark neutrinos would entail corroborating signals at neutrino detectors, which have the ability to point to the source of the flux, in our case the Galactic center. This is an advantage when searching for electrically neutral particles such as neutrinos and photons that are produced in the annihilation of dark matter, for the propagation of charged particles would require additional astrophysical input such as the effect of magnetic fields, emission of diffuse gamma rays and synchrotron radiation, and so on.

Dark neutrino fluxes arise naturally in neutrino portal dark matter models [21-23]. When interpreting our constraints in terms of this setup, parametric regions that could potentially explain the "missing satellites" problem of structure formation can be probed extensively. In these models the local nonrelativistic population of dark matter itself scatters with nucleons in direct detection experiments; however this proceeds through a loop-induced coupling to the $Z$ boson, and the rate is suppressed. Thus, this is an example of a theory where direct detection could find dark matter not so directly, but rather by detecting its "friends" such as its annihilation products. Such a detection scenario is also a generic prediction of the assisted freeze-out mechanism [24]. In this framework, dark matter maintains thermal equilibrium with the primordial plasma even though its annihilation products do not belong to the SM, but rather scatter efficiently with SM states. This possibility has given rise to a growing literature on the prospects of laboratory detection of these annihilation products, called "boosted dark matter" [20,25-40]. While most of these efforts have focused on signals at neutrino experiments, a few such as $[20,35,39]$ have also focused on discovery at direct detection experiments.

Reference [20] in particular explored boosted dark matter with nucleophilic couplings, producing elastic nuclear recoils in direct detectors. We will interpret our flux sensitivities in terms of this scenario as well. We will first show that bounds from proton recoils in the liquid scintillator neutrino detector BOREXINO already outperformed those of Ref. [20] from LUX, for dark matter masses of 100-1000 MeV. Then we show that for present-day annihilations of dark matter with a thermal cross section, couplings as weak as the weak interaction may be probed by DARWIN and ARGO, improving on Ref. [20]'s limit on the couplings by a factor of $\sim 500$. We also argue that detecting boosted dark matter with tiny baryonic couplings may hint at a solution to the "corecusp" problem and show the relevant parameter space that may be probed.

This paper is laid out as follows. In Sec. II, we derive the fluxes and event rates, including a careful treatment of backgrounds from atmospheric and diffuse supernova neutrinos, and nuclear recoil acceptances essential for rejecting electron recoil backgrounds from solar neutrinos. We then translate these to projected sensitivities and compare with bounds at neutrino detectors such as Super-Kamiokande. In Sec. III, we interpret these results in terms of the neutrino portal model and boosted dark matter. We conclude and discuss future possibilities in Sec. IV.

\section{FLUXES, SIGNAL RATES, AND SENSITIVITIES}

In this section we estimate the flux of dark neutrinos and boosted dark matter from Galactic and extragalactic annihilations of dark matter, which we will use to obtain scattering rates at xenon and argon detectors. We then derive the sensitivities to these fluxes at DARWIN and ARGO, accounting for realistic event acceptances and irreducible neutrino backgrounds. We compare these with bounds from scattering on nucleons at XENON1T and BOREXINO, and from other processes at neutrino experiments.

\section{A. Fluxes}

The differential flux of dark neutrinos or boosted dark matter from Galactic annihilations is given by

$$
\frac{d^{2} \Phi}{d \Delta \Omega d E_{\nu}}=\eta \frac{r_{\odot}}{4 \pi}\left(\frac{\rho_{\mathrm{DM}, \odot}}{m_{\mathrm{DM}}}\right)^{2} \mathcal{J}_{\mathrm{ann}} \frac{\left\langle\sigma_{\mathrm{ann}} v\right\rangle}{2} \frac{d N}{d E_{\nu}}
$$

where $\eta=1 / 2$ accounts for dark matter not being selfconjugate, $r_{\odot}=8.33 \mathrm{kpc}$ is the distance of the Sun from the Galactic center, the $(4 \pi)^{-1}$ accounts for isotropic emission, $\rho_{\mathrm{DM}, \odot}=0.4 \mathrm{GeV} / \mathrm{cm}^{3}$ is the dark matter density at the solar position [41], $m_{\mathrm{DM}}$ is the dark matter mass, $\left\langle\sigma_{\mathrm{ann}} v\right\rangle$ is the thermally averaged annihilation cross section of dark matter, and $d N / d E_{\nu}=2 \delta\left(E_{\nu}-m_{\mathrm{DM}}\right)$ is the (monochromatic) energy spectrum of dark neutrinos or boosted dark matter. The dimensionless factor $\mathcal{J}_{\text {ann }}$ accounts for integrating over the dark matter distribution in the line of sight for an angular direction, 


$$
\mathcal{J}_{\text {ann }}=\frac{1}{r_{\odot} \rho_{\mathrm{DM}, \odot}^{2}} \int_{\text {l.o.s. }} \rho_{\mathrm{DM}}^{2}\left(\sqrt{r_{\odot}^{2}-2 s r_{\odot} \cos \theta+s^{2}}\right) d s .
$$

We use the interpolation functions for $\mathcal{J}_{\text {ann }}$ provided in [42] to integrate over the $4 \pi$ sky and obtain for the NavarroFrenk-White halo profile ${ }^{1}[44]$,

$$
\Phi=5.6 \times 10^{-2} \mathrm{~cm}^{-2} \mathrm{~s}^{-1}\left(\frac{\left\langle\sigma_{\mathrm{ann}} v\right\rangle}{10^{-25} \mathrm{~cm}^{3} / \mathrm{s}}\right)\left(\frac{100 \mathrm{MeV}}{m_{\mathrm{DM}}}\right)^{2},
$$

where we have normalized $\left\langle\sigma_{\mathrm{ann}} v\right\rangle$ to the thermal cross section in this range of dark matter masses [45].

Anticipating an interpretation of our results in terms of a model where dark matter couples dominantly to the tau neutrino, we will display our main results assuming that dark neutrinos are produced exclusively through the channel $\chi \bar{\chi} \rightarrow \nu_{\tau} \bar{\nu}_{\tau}$. We will also briefly discuss the possibility that dark matter annihilates to neutrino mass eigenstates, $\chi \bar{\chi} \rightarrow \nu_{i} \bar{\nu}_{i}(i=1,2,3)$ with branching fractions proportional to the squared neutrino masses. This could happen, e.g., in models where neutrino masses are generated by the breaking of lepton number symmetry. In either scenario, the flavor content of the dark neutrinos after propagation through astrophysical distances and arrival at Earth is irrelevant for coherent scattering at direct detection experiments. It would, however, be important for neutrino experiments, where searches depend on the flavor of the neutrinos and/or antineutrinos being detected.

The total flux of dark neutrinos or boosted dark matter could also receive a contribution from dark matter annihilations in extragalactic sources. These may be divided into unclustered and clustered populations. The unclustered contribution is trivially negligible: the (cosmic density $)^{2}$ of dark matter is $\sim 10^{-10} \times$ the (local density) $)^{2}$. The exact contribution of the clustered population depends sensitively on the astrophysical modeling of enhancement factors from halo substructure. For instance, using the redshift-dependent enhancement factors in [46], we find that the extragalactic flux is $\mathcal{O}(10)$ smaller than the Milky Way flux for the case of noninteracting dark matter. For the case of dark matter interacting strongly with dark neutrinos, a scenario that we consider in Sec. III A, Ref. [46] finds that this flux is smaller by one more order of magnitude.

However, Ref. [47] has determined that the extragalactic flux is comparable to the Galactic one, by using different models of substructure enhancement. In what follows, we will show the possible sensitivities of our detectors obtainable from such a flux. To do so, we simply rescale our Galactic flux in Eq. (2) with the total

\footnotetext{
${ }^{1}$ Using a different halo profile could change the flux slightly, e.g., a cored Burkert profile that is shown to be consistent with Milky Way data [43], gives a $4 \pi$-sky flux that is $\simeq 0.55$ times smaller than the NFW profile.
}

(Galactic + extragalactic) flux presented in [47]. This approach neglects redshifts in momenta, i.e., the annihilation products are still taken as monochromatic. For this reason we do not include the extragalactic flux when displaying our main results, and only use it to visualize our optimistic sensitivities.

Contributions to the flux may also stem from dark matter density enhancements around intermediate mass black holes [48-52], but we will not pursue this possibility.

\section{B. Coherent scattering rates}

We now determine the cross sections and scattering event rates of dark annihilation products at our detectors, treating the cases of dark neutrinos and boosted dark matter simultaneously. In addition to coherent nuclear scattering, dark neutrinos also undergo electron scattering at direct detection; however the rates are many orders of magnitude smaller (see, e.g., [10]), and therefore we will not consider electron recoil signals in our study.

For a target nucleus with mass $m_{\mathrm{T}}, N$ neutrons and $Z$ protons, the differential coherent scattering cross section is given by [53]

$$
\frac{d \sigma}{d E_{\mathrm{R}}}\left(E_{\nu}, E_{\mathrm{R}}\right)=\frac{G_{\mathrm{T}}^{2}}{4 \pi} Q_{\mathrm{W}}^{2} m_{\mathrm{T}}\left(1-\frac{m_{\mathrm{T}} E_{\mathrm{R}}}{2 E_{\nu}^{2}}\right) F^{2}\left(E_{\mathrm{R}}\right)
$$

where $Q_{\mathrm{W}}=N-\left(1-4 \sin ^{2} \theta_{\mathrm{W}}\right) Z$, with $\sin ^{2} \theta_{\mathrm{W}}=0.2387$ the weak mixing angle at low energies [54], and $G_{\mathrm{T}}$ is a coupling strength that depends on both the annihilation product and target element, given by [55]

$G_{\mathrm{T}}= \begin{cases}G_{\mathrm{F}} & \text { for dark neutrinos, } \\ \sqrt{2} G_{\mathrm{B}}\left((N+Z) / Q_{\mathrm{W}}\right) & \text { for boosted dark matter. }\end{cases}$

Here $G_{\mathrm{F}}=1.1664 \times 10^{-5} \mathrm{GeV}^{-2}$ is the Fermi constant, and $G_{\mathrm{B}}$ is an effective baryonic coupling of boosted dark matter, whose origins we spell out in Sec. III B. The above equation implies that, for boosted dark matter, $G_{\mathrm{T}} / G_{\mathrm{B}}=\{2.471$, 2.670,31.288\} for the target nuclei $\left\{{ }_{54}^{132} \mathrm{Xe},{ }_{18}^{40} \mathrm{Ar},{ }_{1}^{1} \mathrm{H}\right\}$.

The nuclear form factor $F\left(E_{\mathrm{R}}\right)$ is best parametrized by the Helm form factor [56] for the momentum transfers we are concerned with and is given by

$$
F\left(E_{\mathrm{R}}\right)=3 \frac{j_{1}\left(q r_{n}\right)}{q r_{n}} e^{-q^{2} s^{2} / 2}
$$

where $j_{1}$ is the spherical Bessel function, $s=0.9 \mathrm{fm}$ is the nuclear skin thickness, $q=\sqrt{2 m_{\mathrm{T}} E_{\mathrm{R}}}$ is the momentum transfer, and $r_{n}=\left(c^{2}+\frac{7}{3} \pi^{2} a^{2}-5 s^{2}\right)^{1 / 2}$ parametrizes the nuclear radius, with $c$ and $a=1.23 A^{1 / 3}-0.6 \mathrm{fm}$ and $0.52 \mathrm{fm}$ respectively.

In the left panel of Fig. 1 we show $d \sigma / d E_{\mathrm{R}}$ versus $E_{\mathrm{R}}$ with xenon and argon targets, for neutrino energies $30 \mathrm{MeV}$ 

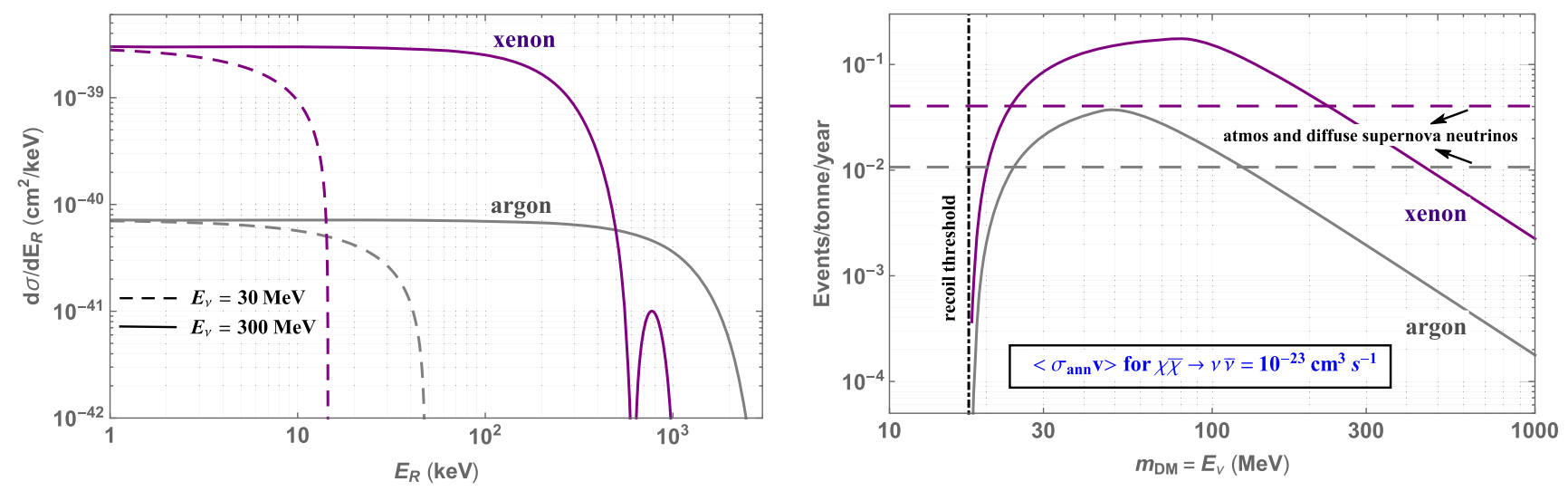

FIG. 1. Left. Differential cross section for coherent scattering of neutrinos with xenon and argon nuclei, for neutrino energies $30 \mathrm{MeV}$ and $300 \mathrm{MeV}$. Argon, being lighter, recoils to higher energies. The effect of the Helm form factor, which suppresses the cross section at high recoil energies, is seen here as a bump in the cross section and is only important at recoil energies $\gtrsim 10^{3} \mathrm{keV}$-which far exceeds the cutoff $\sim 10^{2} \mathrm{keV}$ below which we accept events for our study. Right. The scattering rate of dark neutrinos $\left[G_{\mathrm{T}}=G_{\mathrm{F}}\right.$ in Eq. (3)] per tonne-year of exposure at liquid xenon and argon detectors, assuming dark matter annihilation cross section $\left\langle\sigma_{\text {ann }} v\right\rangle=10^{-23} \mathrm{~cm}^{3} / \mathrm{s}$ and an NFW halo profile. Events are integrated in the recoil energy window $5 \mathrm{keV}-100 \mathrm{keV}$ for xenon and $17 \mathrm{keV}-110 \mathrm{keV}$ for argon; the lower ends of these ranges correspond to neutrino energy $\simeq 17 \mathrm{MeV}$. Also shown for reference is the rate of atmospheric and diffuse supernova neutrinos, which make up our primary irreducible background.

and $300 \mathrm{MeV}$. Note that the maximum recoil energy limited by kinematics is

$$
E_{\mathrm{R}, \max }^{\mathrm{kinem}}=\frac{2 E_{\nu}^{2}}{m_{\mathrm{T}}+2 E_{\nu}} .
$$

The differential scattering rate (per tonne of detector mass) is now obtained from Eqs. (1) and (3) as

$$
\frac{d R}{d E_{\mathrm{R}}}=N_{\mathrm{T}}^{\mathrm{ton}} \int_{E_{\nu, \min }}^{\infty} d E_{\nu} \frac{d \Phi}{d E_{\nu}} \frac{d \sigma}{d E_{\mathrm{R}}},
$$

where $N_{\mathrm{T}}^{\text {ton }}=4.57 \times 10^{28}\left(1.51 \times 10^{27}\right)$ is the number of nuclei per tonne of liquid $\mathrm{Xe}$ ( $\mathrm{Ar}$ ), and $E_{\nu, \text { min }}=\sqrt{m_{\mathrm{T}} E_{\mathrm{R}} / 2}$ is the minimum $E_{\nu}$ required to induce a nuclear recoil of energy $E_{\mathrm{R}}$.

In order to apply the above treatment to future noble liquid detectors, we assume the following fiducial detector masses:

\section{DARWIN : 40 tonnes, \\ ARGO: 300 tonnes.}

The DARWIN mass is as advertised in [5], and the ARGO mass is a realistic possibility [57].

To obtain the total event count at DARWIN, we integrate the rate in Eq. (7) over the range $E_{\mathrm{R}} \in[5 \mathrm{keV}, 100 \mathrm{keV}]$. Below this range solar neutrinos would populate a steep "wall" of background events [58,59]; the upper end of this range is chosen for showing conservative limits. At ARGO, we use the range $E_{\mathrm{R}} \in[17 \mathrm{keV}, 110 \mathrm{keV}]$, where the lower end is once again chosen to evade the solar $\nu$ background, and the upper end is chosen to approximately match with the DARWIN range. The choice of these $E_{\mathrm{R}}$ ranges also ensures that the contribution of inelastic processes (quasielastic scattering, production of resonant states and deep inelastic scattering) to the total event rate is negligible [59].

In the right panel of Fig. 1 we show as a function of $m_{\mathrm{DM}}$ the integrated event rate per tonne-year for scattering of dark neutrinos (i.e., setting $G_{\mathrm{T}}=G_{\mathrm{F}}$ ) with a flux corresponding to $\left\langle\sigma_{\mathrm{ann}} v\right\rangle=10^{-23} \mathrm{~cm}^{3} / \mathrm{s}$. Also shown as horizontal dashed lines are the integrated rates of atmospheric and diffuse supernova neutrino scattering taken from [58], which constitute our main background. The signal rate at argon detectors peaks at lower neutrino energies compared to xenon detectors since $E_{\mathrm{R}, \max }^{\mathrm{kinem}}$ in Eq. (6) attains the maximum $E_{\mathrm{R}}$ imposed by us here at a lower $E_{\nu}$ for argon than for xenon. We will find below that this allows DARWIN and ARGO to probe some regions in complementary ranges of dark matter mass. Figure 1 also shows that the signal rate at xenon detectors is roughly an order of magnitude higher than at argon detectors, implying that the latter require $\sim 10 \times$ the exposure of the former to achieve comparable sensitivities.

\section{Sensitivities and other constraints}

We now obtain the sensitivities for various detector exposures. In a realistic detector, the rejection of electronic recoil (ER) backgrounds comes at the cost of nuclear recoil (NR) acceptance. It was determined by [14] that at DARWIN, where ERS and NRs are distinguished by comparing $S 1$ and $S 2$ scintillation pulses, it is possible to achieve $99.98 \%$ ER rejection with $30 \%-50 \%$ NR acceptance, which is at a level 

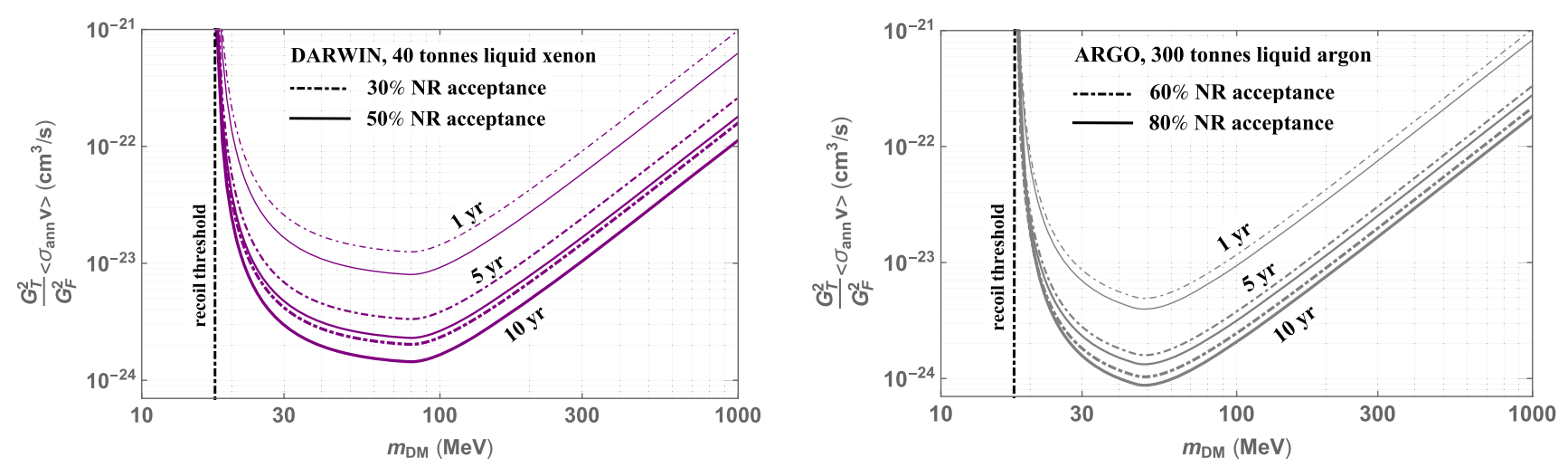

FIG. 2. $90 \%$ C.L. sensitivities of the liquid xenon DARWIN (left) and liquid argon ARGO (right) detectors, with fiducial masses of 40 tonnes and 300 tonnes respectively, to $\left\langle\sigma_{\mathrm{ann}} v\right\rangle \times$ the coupling of the annihilation products to nucleons (normalized to the Fermi constant), as a function of dark matter mass. An NFW halo profile is assumed. The sensitivities are shown for 1, 5 and 10 years of exposure, and for nuclear recoil acceptances of $30 \%$ and $50 \%$ for DARWIN, and $60 \%$ and $80 \%$ for ARGO. The electronic recoil rejection is assumed to be at a level that renders background leakage from solar neutrino-electron scattering negligible. See Sec. II C for more details.

that renders ER backgrounds negligible. This study was performed with $E_{\mathrm{R}} \in[5 \mathrm{keV}, 20.5 \mathrm{keV}]$ to optimize for signal vs background for light WIMPs, which have rapidly falling recoil spectra. Our signal spectra fall much more slowly (Fig. 1), and we use $E_{\mathrm{R}} \in[5 \mathrm{keV}, 100 \mathrm{keV}]$; nevertheless we adopt the above NR acceptances to set our future limits. At liquid argon detectors, which use the pulse shape discrimination technique to reject ER backgrounds, the NRS are better distinguished, yielding higher NR acceptances. We assume that acceptances at the level of 60\%-80\% would be achieved at ARGO [57] with an ER rejection rate that renders ER backgrounds negligible.

We next assume that atmospheric and diffuse supernova neutrinos constitute our sole background. With the above NR acceptances (which we denote by $\epsilon_{\mathrm{NR}}$ ) at DARWIN and ARGO, we may safely neglect the leakage of solar neutrinoelectron scattering events into our neutrino-nucleus scattering regions. The number of signal and background events at a detector before and after accounting for the NR acceptance is simply related by $S_{\mathrm{acc}}=\epsilon_{\mathrm{NR}} S, B_{\mathrm{acc}}=\epsilon_{\mathrm{NR}} B$. Using Poisson statistics, the $90 \%$ C.L. bound is then obtained by solving

$$
\frac{\Gamma\left(B_{\mathrm{acc}}+1, S_{\mathrm{acc}}+B_{\mathrm{acc}}\right)}{B_{\mathrm{acc}} !}=0.1,
$$

where $\Gamma$ is the incomplete gamma function. This allows us to set $\epsilon_{\mathrm{NR}}$-dependent bounds on the flux of dark neutrinos and boosted dark matter.

In Fig. 2 we show the sensitivity of DARWIN and ARGO after exposure times of 1,5 , and 10 years. We show this as a function of dark matter mass for the quantity $\left(G_{\mathrm{T}}^{2} / G_{\mathrm{F}}^{2}\right)\left\langle\sigma_{\mathrm{ann}} v\right\rangle$, which collects the unknown parameters in Eq. (7). The solid and dashed curves indicate NR acceptances of $50 \%$ and $30 \%$ respectively at DARWIN, and $80 \%$ and $60 \%$ respectively at ARGO. As expected, due to the atmospheric and diffuse supernova neutrino background, the sensitivities do not improve linearly with the exposure.

In the left panel of Fig. 3 we show the 10-year DARWIN and ARGO sensitivities with $50 \%$ and $80 \%$ NR acceptances respectively, along with sensitivities from a possible extragalactic flux obtained by the rescaling procedure described in Sec. II A. It must be noted that the true sensitivity lies somewhere between these curves due to the nonmonochromatic nature of the extragalactic neutrino flux. Due to the effect of the maximum $E_{\mathrm{R}}$ imposed by kinematics and our range of integration (see Sec. II B), these two detectors would probe small cross sections in complementary $m_{\mathrm{DM}}$ ranges. We also show with red curves the sensitivity of current-generation liquid xenon detectors (e.g., XENON1T, LUX, PandaX), if operated over the same recoil energy range as DARWIN, after 1 tonne-year of exposure. Recently XENON1T conducted a search for WIMPs with this exposure [60] in nuclear recoil energies $E_{\mathrm{R}} \in[4.9 \mathrm{keV}, 40.9 \mathrm{keV}]$, with a signal selection efficiency of $85 \%$ and with an ER-induced NR background of two events. The NR selection efficiency after ER-discrimination is not quoted, but one expects this to be near $50 \%$ for xenon TPCs (as reflected in the projection for DARWIN). For deriving our sensitivity, we take the range $E_{\mathrm{R}} \in[5 \mathrm{keV}$, $100 \mathrm{keV}$ ] to compare with DARWIN, 50\% signal selection, and no backgrounds. These choices make our estimated sensitivity slightly stronger than the actual bound one would obtain from recasting the XENON1T wIMP search. Lastly, we also show with green curves bounds from measurements of harder-than-solar neutrinos by the liquid scintillator neutrino experiment BOREXINO [61]. In the Appendix B we describe the details of this bound, including the method used for extracting information about dark matter-proton scattering from electron recoil energies. BOREXINO constrains the dark neutrino flux worse than 

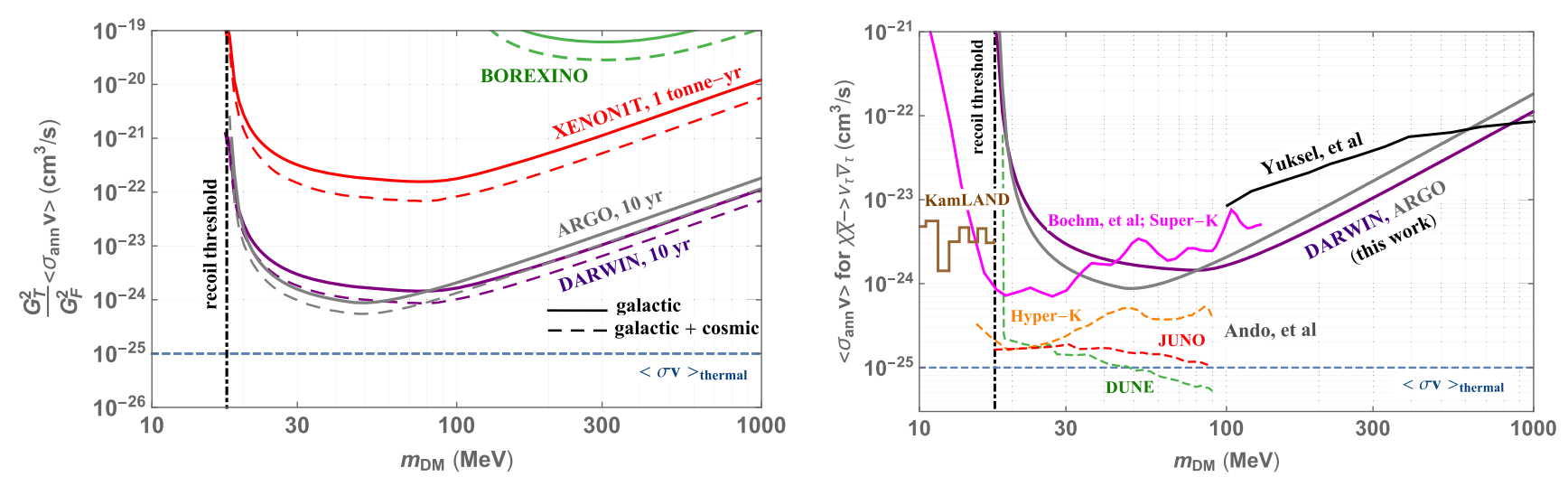

FIG. 3. Left. Same sensitivities as Fig. 2, of the detectors DARWIN (with 10 years of run-time and 50\% NR acceptance), ARGO (with 10 years of run-time and $80 \%$ NR acceptance), and XENON1T after 1 tonne-year of exposure. Also shown is the bound from proton recoils in BOREXINO (derived in Appendix B). For all these bounds an NFW halo profile is assumed. These are compared with sensitivities from a possible extragalactic flux, which we compute using the approximate method described in Sec. II A. Right. Comparison of DARWIN and ARGO sensitivities (as shown in the left panel) to a Galactic flux of dark neutrinos from the channel $\chi \bar{\chi} \rightarrow \nu_{\tau} \bar{\nu}_{\tau}$, with current and future neutrino detectors. Direct detection would surpass the bound from Super-K relic neutrino searches [9] and that derived by Yuksel, et al. [2] in the dark matter mass range $35 \mathrm{MeV}-800 \mathrm{MeV}$. If dark neutrinos are produced instead as mass eigenstates in majoronmediated dark matter annihilations, $\chi \bar{\chi} \rightarrow \nu_{i} \bar{\nu}_{i}(i=1,2,3)$, the bounds from neutrino experiments would be weakened by an $\mathcal{O}(10)$ factor while the DARWIN and ARGO sensitivities are not affected. See Sec. II C for more details.

XENON1T, but constrains boosted dark matter at comparable and stronger levels, as we will see in Sec. III B.

In the right panel of Fig. 3 we show our sensitivities to the annihilation cross section of $\chi \bar{\chi} \rightarrow \nu_{\tau} \bar{\nu}_{\tau}$ by setting $G_{\mathrm{T}}=G_{\mathrm{F}}$. Strictly speaking, of course, this sensitivity must be multiplied by the branching fraction into this annihilation channel (taken here to be $100 \%$ ). The dark neutrino flux is assumed to originate from dark matter in the Milky Way alone. For DARWIN, we assume 50\% NR acceptance, and for ARGO, 80\% NR acceptance. These are compared with bounds from neutrino experiments. The brown curve is obtained from a $90 \%$ C.L. limit set by the liquid scintillator detector experiment KamLAND [62] on extraterrestrial $\bar{\nu}_{e}$ fluxes in the $8.3-18.3 \mathrm{MeV}$ energy range. ${ }^{2}$ The magenta curve is the $90 \%$ C.L. limit, as recast by [9], from a search for diffuse supernova neutrinos by the water Ĉerenkov detector Super-Kamiokande [64] using 2853 days of data. The relevant search channels are inverse beta decay $\left(\bar{\nu}_{e}+p \rightarrow e^{+}+n\right)$ and absorption in oxygen $\left(\nu_{e} / \bar{\nu}_{e}+{ }^{16} \mathrm{O} \rightarrow e^{ \pm}+X\right)$, sought in the positron energy range 16-88 MeV, which allows for probing $E_{\nu} \leq$ $130 \mathrm{MeV}$ [9].

The dashed curves are 90\% C.L. sensitivities, as estimated by [47], of the future neutrino detectors HyperKamiokande, DUNE, and JUNO. The search channels at the water Ĉerenkov detector Hyper-K are the same as Super-K

\footnotetext{
${ }^{2}$ The neutrino flux in this energy range can also be potentially bounded by dark matter direct detection searches that look for electronic recoils in ionization signals from nuclear recoils [63]; however, the uncertainties are too large for these searches to compete with dedicated neutrino experiments such as KamLAND.
}

above; at the liquid argon detector DUNE it is the charged current process $\nu_{e} / \bar{\nu}_{e}+{ }^{40} \mathrm{Ar} \rightarrow e^{ \pm}+\{X\}$ (where $\{X\}$ are nuclei); and at the liquid scintillator detector JUNO these are inverse beta decay and absorption in carbon $\left(\bar{\nu}_{e}+{ }^{12} \mathrm{C} \rightarrow e^{+}+{ }^{12} \mathrm{~B}\right.$ and $\left.\nu_{e}+{ }^{12} \mathrm{C} \rightarrow e^{-}+{ }^{12} \mathrm{~N}\right)$. For this study a run-time of 3000 days was assumed, and the backgrounds were estimated by rescaling those of the Super-K analysis above; these sensitivities also depend on the energy resolution assumed for each detector. Finally, the black curve is the bound (with unspecified C.L.), as derived by Yuksel, et al. [2], from atmospheric neutrino measurements by Super-K, Fréjus, and AMANDA.

For the neutrino experiment bounds, we accounted for the fact that the signal flux for Dirac dark matter is halved compared to self-conjugate dark matter. Moreover, for the reaches of the future detectors Hyper-K, DUNE and JUNO we rescaled the total fluxes used in [47] by the method described in Sec. II A to account for dark matter annihilations in the Milky Way alone.

We see in Fig. 3 that DARWIN and ARGO would rival, even outdo, the bounds from Super-K diffuse neutrino searches (Boehm, et al.) and atmospheric neutrino measurements (Yuksel, et al.). This is because, as mentioned in the Introduction, though large-volume neutrino detectors operate at greater exposures than dark matter detectors, the latter would compensate via the higher event rates of coherent nuclear scattering.

Furthermore, the neutrino detector bounds depend on the fraction of the dark neutrino flux that is electron-flavored. In the case of $\chi \bar{\chi} \rightarrow \nu_{\tau} \bar{\nu}_{\tau}$, the propagation of neutrinos over galactic distances washes out coherent oscillations, so that this fraction is simply the combined electron component of the mass eigenstates produced at the source. We calculate 
this fraction in Appendix A, finding that the $e: \mu: \tau$ flavor ratio upon arrival at Earth is 11: 19: 20, i.e., the electron fraction is 0.22 . We have accounted for this reduction of effective flux when presenting our neutrino experiment bounds. Another interesting case is that of dark matter annihilating directly into neutrino mass eigenstates, $\chi \bar{\chi} \rightarrow \nu_{i} \bar{\nu}_{i}$, so that no oscillations occur. In theories where neutrinos acquire masses through lepton number breaking (see [65] and references therein), such annihilations are mediated in the $s$-channel by the majoron, the pseudoscalar associated with the symmetry breaking, which couples to the mass eigenstates in proportion to the neutrino eigenmass $m_{\nu, i}{ }^{3}$. Then the branching fractions into $\nu_{i} \bar{\nu}_{i}$ are proportional to $m_{\nu, i}^{2}$. In this case, assuming normal hierarchy of neutrino masses we find that the flavor ratio upon arrival at Earth is 3: 52: 45 (see Appendix A), so that the electron fraction of the flux is 0.03 . A scenario such as this would therefore weaken the bounds from neutrino experiments shown in Fig. 3 by an order of magnitude, so that direct detection bounds are far stronger.

While we have shown the reaches of future neutrino experiments for energies $\lesssim 100 \mathrm{MeV}$, there are no existing studies on these reaches for energies between $100 \mathrm{MeV}$ and $1 \mathrm{GeV}$. The exact sensitivities should depend on backgrounds from atmospheric neutrinos, and the systematics and energy resolutions of these detectors in this energy range. Estimating these is beyond the scope of our work; however, on the strength of the reaches in the sub-100 MeV range, it may be surmised that the reaches in the $>100 \mathrm{MeV}$ energy range may be somewhat stronger than DARWIN and ARGO in most regions for the case of tauflavored dark neutrinos. For the case of majoron-mediated dark neutrinos discussed above, dark matter direct detection reaches may still be stronger in most regions. To make matters even more interesting, the liquid scintillator detector JUNO may see proton recoils from dark neutrinos that are not electron-flavored, à la BOREXINO as discussed above. Therefore, in the event of discovery, an intricate interplay of dark matter and neutrino experiments will be required to discern whether dark neutrinos are produced in flavor or mass eigenstates.

We end this subsection by noting that the smallest annihilation cross sections that may be probed by direct detection experiments would exceed $10^{-25} \mathrm{~cm}^{3} / \mathrm{s}$, the value required for obtaining the observed dark matter relic abundance $\left(\Omega_{\chi} h^{2}=0.12\right)$ via thermal freeze-out. If a dark neutrino flux is detected at DARWIN and ARGO, this would

\footnotetext{
${ }^{3}$ Obtaining a large annihilation cross section in this scenario is a model-building challenge that is beyond the scope of our work. An interesting related possibility is a "secluded" scenario [66] where the dark matter annihilates with a large cross section to a pair of on shell mediators which then each decay dominantly to $\nu_{3} \bar{\nu}_{3}$. Such neutrinos are no longer monochromatic, yielding interesting signatures at direct detection and neutrino experiments.
}

imply that dark matter acquired the observed abundance through a nonstandard cosmological history, such as, e.g., late decays of long-lived states into a dark matter population.

\section{Distinguishing between dark neutrinos and boosted dark matter}

If a novel flux of coherently scattering particles is discovered at dark matter direct detection searches, it would be of vital importance to identify the species detected. As said in the Introduction, dark neutrinos may be distinguished from nonrelativistic WIMPs and boosted dark matter if corroborating, directional signals are obtained at neutrino experiments. We now show that it is in principle possible to distinguish between dark neutrinos and boosted dark matter as well, given sufficient statistics.

First we note that there is a degeneracy among the unknown parameters $G_{\mathrm{T}},\left\langle\sigma_{\mathrm{ann}} v\right\rangle$ and $m_{\mathrm{DM}}$ in the scattering rate, Eq. (7). If both DARWIN and ARGO see positive signals, then $\left\langle\sigma_{\mathrm{ann}} v\right\rangle$ may be eliminated in the ratio of integrated rates at either detector, Events DARWIN $_{\text {Events }}$ ARGO $_{\text {. Now }}$ we recall that $G_{\mathrm{T}}$ is species-dependent [Eq. (4)], since neutrinos scatter preferentially on neutrons whereas boosted dark matter scatters democratically on both neutrons and protons, as well as is target-dependent, since xenon and argon nuclei comprise different nucleon populations. Thus Events DARWIN $_{\text {/Events }}$ ARGO would distinguish between dark neutrinos and boosted dark matter for a given projectile energy (= dark matter mass). We have plotted this quantity (with the backgrounds subtracted) for the two species in Fig. 4, as a function of $m_{\mathrm{DM}}$. The scattering rates have been integrated over the $E_{\mathrm{R}}$ ranges

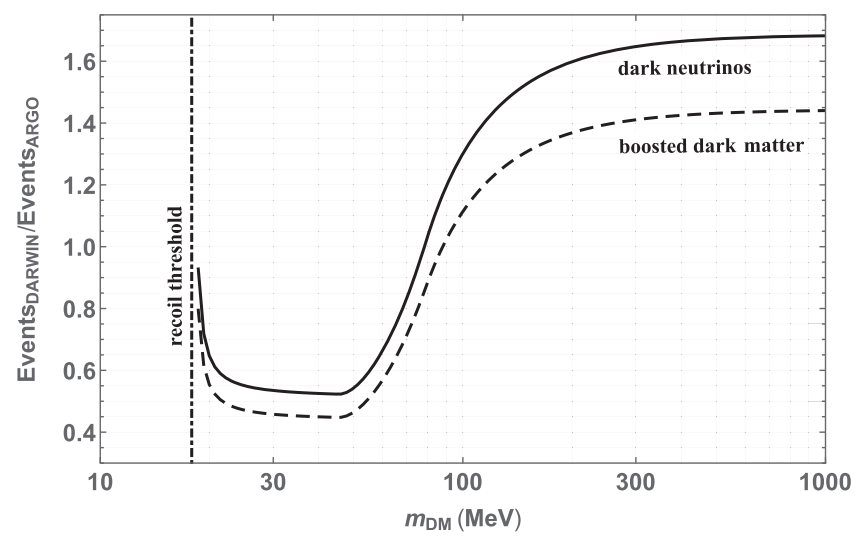

FIG. 4. A simple diagnostic to distinguish between dark neutrinos and boosted dark matter, as described in Sec. II D. Shown here is the ratio of background-subtracted events at DARWIN and ARGO after equal run-times, as a function of dark matter mass. Since neutrinos scatter preferentially on neutrons whereas boosted dark matter scatters equally on neutrons and protons, and since xenon and argon contain different numbers of nucleons, this ratio separates the two cases. 
mentioned in Sec. II B, and equal run-times at both experiments are assumed. The remaining degeneracy, that of dark matter mass, may be broken by inspecting the recoil spectrum at high energies, as the kinematic endpoint is set by this mass [Eq. (6)].

We point out another virtue in simultaneously inspecting recoil spectra at DARWIN and ARGO. If a dark neutrino flux is detected with a small number of signal events, the background model used for the atmospheric and diffuse supernova fluxes would come into question, in which case such an inspection would prove valuable. A detailed statistical study characterizing signals at various detector materials is an exercise we reserve for future exploration.

\section{INTERPRETATIONS}

In this section, we interpret our model-independent results in the previous section in terms of a model of dark matter that interacts with the SM through a neutrino portal, and models of nucleophilic boosted dark matter.

\section{A. Neutrino portal dark matter}

Dark matter annihilation into neutrinos is a generic feature of simple neutrino portal dark matter models [21-23]. In such a setup, dark matter couples to the SM through the operator $H L$, where $H$ is the SM Higgs doublet and $L$ is a lepton doublet containing the neutrino $\nu_{L}$. Taking dark matter to be a Dirac fermion $\chi$, stabilizing it requires charging it under, e.g., a $\mathbb{Z}_{2}$ symmetry, and therefore, coupling it to $H L$ requires introducing a charged complex scalar $\phi$ heavier $^{4}$ than $\chi$. Then the dark matter couples to neutrinos via the effective operator $\phi \bar{\chi} H L / \Lambda \rightarrow(v / \Lambda) \phi \bar{\chi} \nu$ under electroweak symmetry breaking, where $\langle\mathrm{H}\rangle=v=$ $174 \mathrm{GeV}$ and $\Lambda$ is some high scale. In the UV completion, these interactions can arise from a Dirac sterile neutrino $N$ that couples to both the SM and "dark" sectors. The relevant interactions are contained in

$$
\begin{aligned}
-\delta \mathcal{L}= & m_{N} \bar{N} N+m_{\mathrm{DM}} \bar{\chi} \chi+m_{\phi}^{2}|\phi|^{2} \\
& +\left[\lambda_{\ell} \bar{L}_{\ell} i \tau^{2} H^{*} N_{R}+\phi \bar{\chi}\left(y_{L} N_{L}+y_{R} N_{R}\right)+\text { H.c. }\right] .
\end{aligned}
$$

Note that we can assign a lepton number to $N$ and $\chi$ or $\phi$ such that it remains conserved in $\delta \mathcal{L}$. Here $\ell$ is a flavor index. If we assume that the coupling of one flavor is dominant, then the linear combination $\nu \equiv$ $\sqrt{1-\left|U_{\ell 4}\right|^{2}} \nu_{L \ell}-U_{\ell 4} N_{L}$ forms a light (and at this level, massless) neutrino that couples to dark matter through the interaction

\footnotetext{
${ }^{4}$ It is also possible to have $\phi$ lighter than $\chi$, so that $\phi$ is now the dark matter and $\chi$ the mediator. However, the dark matter annihilation proceeds in the $p$-wave, so that annihilation signals today are absent.
}

$$
-\mathcal{L}_{\text {int }}=y_{L} U_{\ell 4} \phi \bar{\chi} \nu+\text { H.c. }
$$

The orthogonal combination pairs up with $N_{R}$ to form a heavy Dirac neutrino with mass $M=\sqrt{\lambda_{\ell}^{2} v^{2}+m_{N}^{2}}$. The active-sterile mixing angle is $U_{\ell 4}=\lambda_{\ell} v / M$. Note that this mixing angle need not be tiny for the mostly active neutrino to be extremely light. ${ }^{5}$

We assume that $m_{\mathrm{DM}}<m_{N} / 2$ so that it can only annihilate to mostly active neutrinos, $\chi \bar{\chi} \rightarrow \nu \bar{\nu}$. In this case, the heavy neutrino decay mode $N \rightarrow \chi \bar{\chi} \nu$ is fully invisible, implying that the limits on the mixing angle $U_{\ell 4}$ can be relatively weak, especially in the case that the dominant mixing is with the $\tau$ neutrino. Focusing on this possibility, the dark matter annihilation cross section can be written

$$
\left\langle\sigma_{\mathrm{ann}} v\right\rangle=\frac{Y_{\tau}}{32 \pi m_{\mathrm{DM}}^{2}}\left(1+\frac{m_{\mathrm{DM}}^{2}}{m_{\phi}^{2}}\right)^{-2},
$$

with

$$
Y_{\tau} \equiv y_{L}^{4}\left|U_{\tau 4}\right|^{4} \frac{m_{\mathrm{DM}}^{4}}{m_{\phi}^{4}}
$$

Comprehensive bounds on this effective coupling have been derived in Ref. [23].

A large mixing angle, or equivalently large effective coupling $Y_{\tau}$, can have interesting implications for structure formation. The interaction of the dark matter and the light neutrinos can be large enough to delay the onset of the growth of structure [21,67-71], suppressing the number of structures with mass below some critical scale, $M_{\text {cut }}$, determined by $Y_{\tau}$. This has been cited as a potential solution to the so-called "missing satellites problem". Despite the large coupling needed to affect structure formation, probing this region of parameter space in other physical systems is difficult, largely due to the challenge of making and detecting $\tau$ neutrinos.

In Fig. 5, we show as a function of $m_{\mathrm{DM}}$ the sensitivities of DARWIN and ARGO to the effective coupling $Y_{\tau}$, which controls dark matter annihilation. We assume 10 year exposures and the NR acceptances used in Fig. 3. Also shown are the bounds from XENON1T and BOREXINO as in the left panel of Fig. 3. Only the Galactic flux is included. In addition, we show with dashed black lines the regions of parameter space that result in $M_{\text {cut }}=10^{7} M_{\odot}$ and $10^{9} M_{\odot}$, given by [23]

$$
M_{\mathrm{cut}} \simeq 10^{8} M_{\odot}\left(\frac{0.1 \mathrm{GeV}}{m_{\mathrm{DM}}}\right)^{-7 / 2} Y_{\tau}^{3 / 4} .
$$

\footnotetext{
${ }^{5}$ Simple extensions to the model can confer finite masses to the light neutrinos while keeping the mixing angle relatively large; see [23] for details.
} 


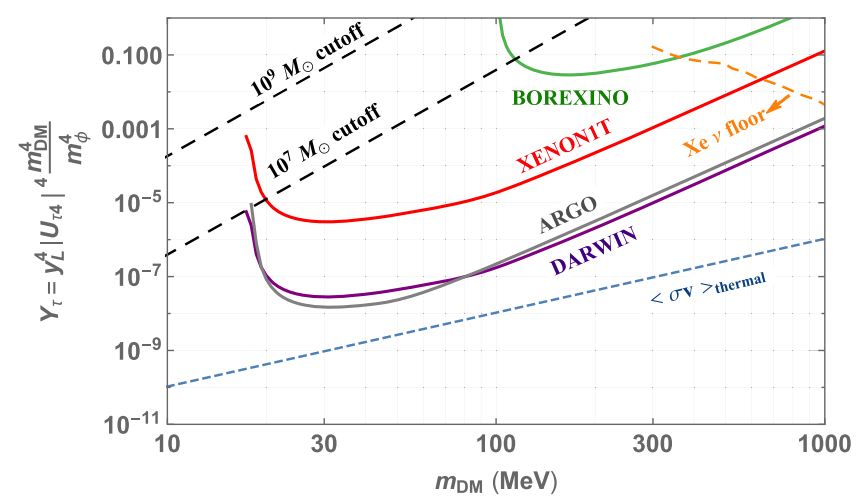

FIG. 5. Interpretation of our results in the left panel of Fig. 3 in terms of the neutrino portal dark matter model described in Sec. III A. Bounds are shown in the plane of the effective coupling $Y_{\tau}$ [Eq. (12)] vs dark matter mass, with regions above the curves excluded. Dashed black curves indicate parameters that result in suppression of the growth of structures below a mass cutoff of $10^{9}$ and $10^{7}$ solar masses, thus explaining the "missing satellites" problem. These regions are seen to be extensively probed by direct detection. The dashed orange curve denotes parameters that correspond to a dark matter-nucleon scattering cross section associated with the standard "neutrino floor" in xenon. The dashed blue line corresponds to a thermal annihilation cross section $=10^{-25} \mathrm{~cm}^{3} / \mathrm{s}$.

We find that direct detection of dark neutrinos could extensively probe these regions. For reference, we also show the value of $Y_{\tau}$ that yields a thermal annihilation cross section of $\left\langle\sigma_{\mathrm{ann}} v\right\rangle=10^{-25} \mathrm{~cm}^{3} / \mathrm{s}$.

As discussed in the Introduction, in this model dark matter develops an effective loop-induced coupling to the $Z$ boson, through which it could scatter with nuclei at direct detection experiments. In Fig. 5 we show with a dashed orange curve values of the coupling $Y$ that correspond to this scattering cross section at the xenon neutrino floor, as done in [23]. In regions above this curve, an interesting possibility may occur: direct detection experiments may register events from coherent nuclear scattering of both the local, nonrelativistic dark matter and the relativistic dark neutrino fluxes. However these regions are already disfavored by the atmospheric neutrino bounds of Yuksel, et al. (see Fig. 3).

Finally, we point to the existence of other dark matter models that would result in monochromatic dark neutrinos. For example, such dark matter could be supersymmetric sneutrinos [52] or an adjoint fermion in a hidden confining gauge group [72]. For variations on the neutrino portal model presented in this section, with all possible spins of the dark matter and the mediator, see [9].

\section{B. Boosted dark matter}

We now consider the possibility that the annihilation products of dark matter are a second, subdominant component of dark matter that is nucleophilic. This is realized when the boosted dark matter is a "baryonic neutrino" whose interactions with baryons are mediated by a gauged $U(1)_{\mathrm{B}}$ vector $[19,55,73]$, or when it is a fermion whose interactions with SM fermions are mediated by a scalar that mixes with the Higgs boson [20]. The effective coupling to nucleons is then $G_{\mathrm{B}}=g_{\mathrm{x}} g_{\mathrm{q}} / m_{\mathrm{MED}}^{2}$, where $g_{\mathrm{x}}$ and $g_{\mathrm{q}}$ are the mediator couplings with the boosted dark matter and quarks respectively, and $m_{\mathrm{MED}}$ is the mediator mass. It has been determined that in both these models, $G_{\mathrm{B}} \lesssim$ $7000 G_{\mathrm{F}}$ is experimentally viable [20]. We take the boosted dark matter's mass $\ll m_{\mathrm{DM}}$, and this implies that its population can contribute to the energy density of radiation in the early Universe, constrained by measurements of $N_{\text {eff }}$; however, this contribution is within observed limits [20].

Using Eq. (4) and the results in Fig. 3, we may obtain bounds on $G_{\mathrm{B}}$ for a flux corresponding to a thermal ${ }^{6}$ annihilation cross section $\left\langle\sigma_{\text {ann }} v\right\rangle_{\text {thermal }}=10^{-25} \mathrm{~cm}^{3} / \mathrm{s}$; these are shown in the left panel of Fig. 6 as constraints on the ratio $G_{\mathrm{B}} / G_{\mathrm{F}}$ as a function of dark matter mass. Here we have neglected the attenuation of flux due to passage through the Earth, since the couplings being probed are too small for this effect to be important.

The BOREXINO bounds, which were the weakest in the case of the dark neutrino flux, now outdo XENON1T for dark matter masses $\geq 200 \mathrm{MeV}$. This is because the relative size of the cross sections for scattering with protons vs xenon is much smaller for neutrinos than for boosted dark matter; see Eqs. (3) and (4). The BOREXINO and XENON1T bounds on $G_{\mathrm{B}}$ are also 2 or 3 orders of magnitude stronger than those placed by [20] using a 0.027 tonne-year LUX data set. [We have adapted Ref. [20]'s bound on $\left\langle\sigma_{\mathrm{ann}} v\right\rangle$ for $G_{\mathrm{B}} / G_{\mathrm{F}}=500$ to present the bound on $\left.\left.\left(G_{\mathrm{B}} / G_{\mathrm{F}}\right)\right|_{\text {thermal }}.\right]$

We see that DARWIN and ARGO would improve the sensitivity to $G_{\mathrm{B}}$ by a factor of 10 , probing all the way down to the weak coupling size $1-2 \times G_{\mathrm{F}}$ for dark matter masses $\sim 25-100 \mathrm{MeV}$. This is one of the main results of our paper.

The core-cusp problem. While probing thermal freezeout is of inherent interest, another worthwhile target for direct detection is to probe scenarios that address the small scale structure of the Universe. In Sec. III A, we explored how direct detection signals of dark neutrinos could help address the missing satellites problem. Now we show briefly that direct detection signals could tackle the socalled core-cusp problem as well.

From N-body simulations of noninteracting cold dark matter, we expect its density to rise steeply near the centers of galactic halos, but observations indicate flat density profiles in these regions. Reference [74] posited that if dark

\footnotetext{
${ }^{6}$ Since for thermal annihilation cross sections the detectable values of $G_{\mathrm{B}}$ turn out to be $>G_{\mathrm{F}}$, one may reasonably assume that the boosted annihilation products, by virtue of scattering with nucleons, keep dark matter in thermal equilibrium in the early Universe until freeze-out.
} 

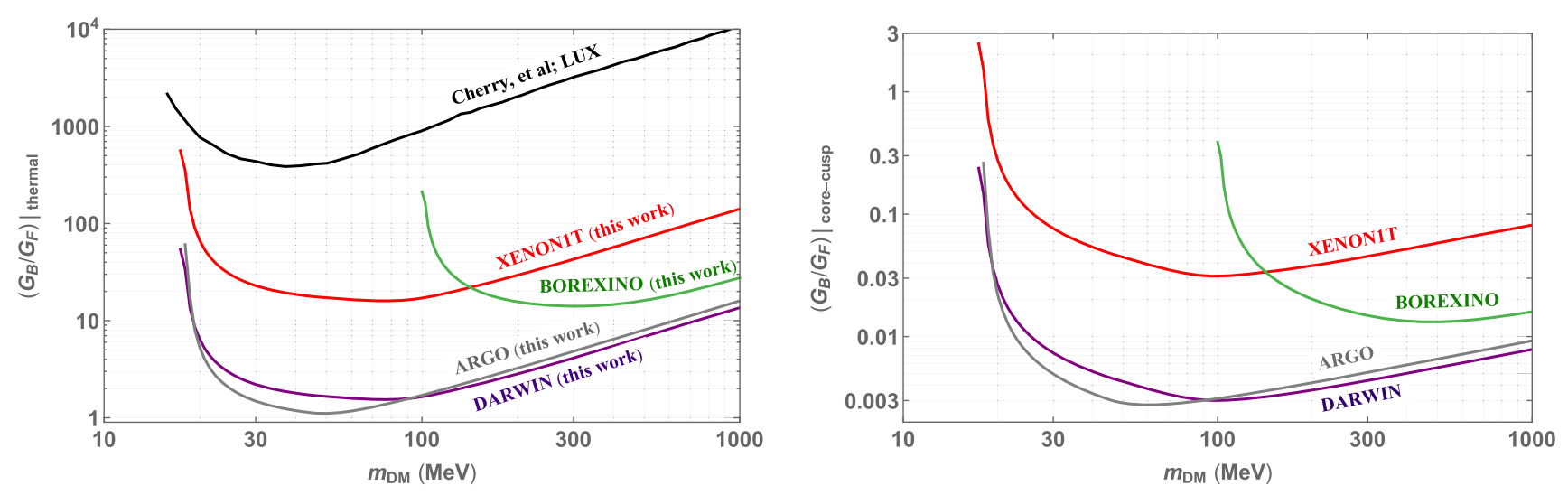

FIG. 6. Interpretation of our constraints on $\left(G_{\mathrm{T}}^{2} / G_{\mathrm{F}}^{2}\right)\left\langle\sigma_{\mathrm{ann}} v\right\rangle$ vs dark matter mass in the left panel of Fig. 3, in terms of the boosted dark matter models described in Sec. III B. Left. Bounds as a function of dark matter mass on the baryonic coupling $G_{\mathrm{B}}$ (normalized to the Fermi constant $G_{\mathrm{F}}$ ) such that the boosted dark matter flux is obtained for a thermal annihilation $\mathrm{cross}^{\mathrm{section}}=10^{-25} \mathrm{~cm}^{3} / \mathrm{s}$. The bounds obtained by Cherry, et al. [20] used LUX data. The erstwhile bound from BOREXINO proton recoils (derived in Appendix B) and the current bound from XENON1T are seen to be stronger. DARWIN and ARGO can be seen to probe $G_{\mathrm{B}}$ all the way down to the weak coupling $G_{\mathrm{F}}$ in the $m_{\mathrm{DM}}$ range $\sim 25-100 \mathrm{MeV}$. Right. Addressing the core-cusp problem with boosted dark matter detection. Shown are values of $G_{\mathrm{B}} / G_{\mathrm{F}}$ ruled out by BOREXINO and XENON1T, and those to be probed by DARWIN and ARGO, for an annihilation cross section [Eq. (14)] that would ensure that dark matter is depleted from halo cores.

matter annihilated today with large rates, it would be especially depopulated in overdense regions, and uniform halo cores would result. The requisite $s$-wave annihilation cross section is far above the thermal cross section for our dark matter masses of interest,

$$
\left\langle\sigma_{\text {ann }} v\right\rangle_{\text {core-cusp }}=3 \times 10^{-20} \mathrm{~cm}^{3} / \mathrm{s}\left(\frac{m_{\mathrm{DM}}}{100 \mathrm{MeV}}\right) .
$$

The annihilation products must not yield photons, since such large $\gamma$ fluxes are well excluded. Nor could the annihilation products be neutrinos, though they are the hardest SM states to detect, as this too is excluded (see Fig. 3). But if the annihilation products are even harder to detect than neutrinos, such as when they are boosted dark matter with tiny couplings to the SM, then a large flux of them could have gone unnoticed, keeping this solution to the core-cusp problem alive. In the right panel of Fig. 6 we show the values of the coupling $G_{\mathrm{B}}$ (relative to $G_{\mathrm{F}}$ ) that are excluded by XENON1T and BOREXINO proton recoils, and probeable by DARWIN and ARGO, if the boosted dark matter flux corresponded to the annihilation cross section in Eq. (14). We see that in this scenario DARWIN and ARGO would be sensitive to couplings $\sim 10^{-3}-10^{-2} \times G_{\mathrm{F}}$ for dark matter masses $\sim 20-1000 \mathrm{MeV}$.

\section{CONCLUSIONS AND DISCUSSION}

In this paper we explored the sensitivity of future noble liquid-based direct detection experiments to the flux of particles produced in the annihilation of dark matter in the sky. Our main results are summarized in Figs. 3, 5, and 6. The particle species we considered are neutrinos, which we call "dark neutrinos", and a second component of dark matter, now commonly known as boosted dark matter, that is nucleophilic. We derived the reaches of a 40-tonne xenon-based detector, DARWIN, and a 300-tonne argonbased detector, ARGO, after accounting for imminent backgrounds from atmospheric and diffuse supernova neutrinos, as well as realistic nuclear recoil acceptances. To the best of our knowledge, this is the first work to study a next-to-next generation liquid argon detector for a concrete theoretical scenario. Liquid argon technology exploits the pulse shape discrimination technique, which is not possible in liquid xenon, and this enables superior electron recoil rejection. We have shown that by virtue of this, ARGO would set limits on dark matter parameters that are closely comparable and complementary to DARWIN. Due to their differing technologies, thresholds and energy ranges of interest, a positive signal at both DARWIN and ARGO would help to mitigate uncertainties in the modeling of backgrounds, especially if signal events are few in number. It would also help to characterize the particle species detected, e.g., neutrinos vs boosted dark matter.

We found that after 10 years of exposure these experiments could probe dark neutrino fluxes beyond the reach of neutrino experiments. There are two main reasons for this: (i) while neutrino detectors admit larger fluxes and sustain greater exposures, direct detection experiments benefit from high event rates due to coherent nuclear scattering, (ii) while search channels at most neutrino experiments such as Super-Kamiokande rely on the $\nu_{e}$ and/or $\bar{\nu}_{e}$ fraction of the dark neutrino flux, which may be as small as $\mathcal{O}\left(10^{-2}\right)$ depending on the dark matter model with which the bounds are interpreted, the search channel of coherent nuclear scattering at direct detection is agnostic to neutrino flavor and self-conjugation. 
We also derived current bounds and sensitivities on the boosted dark matter flux, which are set in complementary dark matter mass ranges by 1 tonne-year of data from XENON1T and by a limit on proton recoils at BOREXINO. For a thermal annihilation cross section, these bounds limit the effective baryonic coupling of the boosted dark matter to $G_{\mathrm{B}} \lesssim(10-100) \times$ the Fermi constant $G_{\mathrm{F}}$; in the future, DARWIN and ARGO would push this down to $G_{\mathrm{B}} \lesssim(1-10) \times G_{\mathrm{F}}$. For an annihilation cross section corresponding to that which is required to deplete dark matter from halo cores, and thus solve the core-cusp problem, DARWIN and ARGO would probe couplings down to $G_{\mathrm{B}} \sim$ $10^{-3}-10^{-2} \times G_{\mathrm{F}}$.

There are several related avenues of exploration that we had not entered in this work. While we had assumed that our signals were sourced by dark matter annihilations in free space, monochromatic fluxes may also originate in annihilations in the Sun, à la Refs. [75] and [76-79]. Nonmonochromatic neutrinos may be sourced by "cascade annihilations", i.e., annihilations of dark matter into mediators or SM states that may subsequently decay to neutrinos in the energy range of interest for direct detection. While we had implicitly assumed a symmetric population of dark matter, an interpretation of our flux limits in terms of an asymmetric population is possible, especially in the case of boosted dark matter, where our limits on the (symmetric) annihilation cross section could be $<10^{-25} \mathrm{~cm}^{3} / \mathrm{s}$ - see [80] for more details. If signals appear in more than one direct detection experiment, a halo-independent analysis for relativistic fluxes may be undertaken, such as in [20]. An exciting possibility is the double signal briefly mentioned in Sec. III A, comprising of the scattering of both local, nonrelativistic dark matter, and relativistic particles produced in dark matter annihilations. While the parametric regions for this to transpire in the simple neutrino portal model we had explored are already excluded, there may be other theories where this is still viable, especially in models of boosted dark matter.

Goodman and Witten [81] originally proposed dark matter direct detection following the proposal of Drukier and Stodolsky [82] for detecting neutrinos-from solar, atmospheric, terrestrial, supernova, reactor, and spallation sources-in coherent elastic neutral current scattering. Should the "ultimate detectors" discover dark matter by catching neutrinos sourced by dark matter, the revolutionary moment would draw a decades-long search program to a satisfying close.

\section{ACKNOWLEDGMENTS}

This work has benefited from conversations with Joe Bramante, Pietro Giampa, David Morrissey, and Tien-Tien Yu. It is supported by the Natural Sciences and Engineering Research Council of Canada (NSERC). TRIUMF receives federal funding via a contribution agreement with the National Research Council Canada.

\section{APPENDIX A: FLAVOR COMPONENTS AFTER NEUTRINO PROPAGATION}

While coherent nuclear scattering at direct detection searches is blind to neutrino flavor, other channels, used in neutrino experiments, are sensitive to the flavor component being detected. These components depend both on the distribution of flavors in the neutrino states produced at the source and neutrino mixing parameters. In this Appendix we calculate these components for the cases of dark matter annihilations into either flavor or mass eigenstates, which we use in Sec. II to present bounds from neutrino experiments.

We begin with the case of $\chi \bar{\chi} \rightarrow \nu_{\alpha} \bar{\nu}_{\alpha}$, where $\alpha$ is a flavor index. Since these dark neutrinos travel galactic distances before arriving at detectors, the effect of coherent oscillations is negligible, so that the probability of detecting flavor $\beta$ is given simply by

$$
P_{\alpha \beta}=\sum_{i=1}^{3}\left|U_{\beta i}\right|^{2}\left|U_{\alpha i}\right|^{2},
$$

where $U$ is the PMNS matrix. It is usually parametrized as

$$
\left[\begin{array}{ccc}
1 & 0 & 0 \\
0 & c_{23} & s_{23} \\
0 & -s_{23} & c_{23}
\end{array}\right]\left[\begin{array}{ccc}
c_{13} & 0 & s_{13} e^{i \delta_{\mathrm{CP}}} \\
0 & 1 & 0 \\
-s_{13} e^{-i \delta_{\mathrm{CP}}} & 0 & c_{13}
\end{array}\right]\left[\begin{array}{ccc}
c_{12} & s_{12} & 0 \\
-s_{12} & c_{12} & 0 \\
0 & 0 & 1
\end{array}\right],
$$

where $c_{a b}$ and $s_{a b}$ denote $\cos \theta_{a b}$ and $\sin \theta_{a b}$ respectively. The most recent best-fit values $[83,84]$ (without the error bars) are

$$
\theta_{12}=33.62^{\circ}, \quad \theta_{23}=47.2^{\circ}, \quad \theta_{13}=8.54^{\circ}, \quad \delta_{\mathrm{CP}}=234^{\circ},
$$

from which we obtain using Eq. (A1) the following conversion probabilities:

$$
\left[\begin{array}{lll}
P_{e e} & P_{e \mu} & P_{e \tau} \\
P_{\mu e} & P_{\mu \mu} & P_{\mu \tau} \\
P_{\tau e} & P_{\tau \mu} & P_{\tau \tau}
\end{array}\right]=\left[\begin{array}{lll}
0.55 & 0.23 & 0.22 \\
0.23 & 0.39 & 0.38 \\
0.22 & 0.38 & 0.40
\end{array}\right]
$$

Next we turn to the case of $\chi \bar{\chi} \rightarrow \nu_{i} \bar{\nu}_{i}$, where $i$ runs over mass eigenstates. Inspired by majoron-mediated models, we assume that the branching fractions $\propto m_{\nu, i}^{2}$. As these neutrinos do not oscillate, the probability of detecting flavor $\alpha$ is

$$
P_{\alpha}=\left(\sum_{i=1}^{3} m_{\nu, i}^{2}\left|U_{\alpha i}\right|^{2}\right) /\left(\sum_{i=1}^{3} m_{\nu, i}^{2}\right) .
$$

Using $\left\{m_{\nu, 1}^{2}, m_{\nu, 2}^{2}, m_{\nu, 3}^{2}\right\}=\left\{0,7.40 \times 10^{-5}, 2.494 \times 10^{-3}\right\} \mathrm{eV}^{2}$, which is consistent with solar and atmospheric neutrino oscillation data $[83,84]$ if a normal mass hierarchy is assumed, we get 


$$
\left[\begin{array}{l}
P_{e} \\
P_{\mu} \\
P_{\tau}
\end{array}\right]_{\text {normal }}=\left[\begin{array}{l}
0.03 \\
0.52 \\
0.45
\end{array}\right]
$$

For an inverted mass hierarchy, $\left\{m_{\nu, 1}^{2}, m_{\nu, 2}^{2}, m_{\nu, 3}^{2}\right\}=$ $\left\{2.42 \times 10^{-3}, 2.494 \times 10^{-3}, 0\right\} \mathrm{eV}^{2}$ is consistent with data, and we get

$$
\left[\begin{array}{l}
P_{e} \\
P_{\mu} \\
P_{\tau}
\end{array}\right]_{\text {inverted }}=\left[\begin{array}{l}
0.48 \\
0.24 \\
0.28
\end{array}\right] .
$$

It is also possible that the neutrino mass spectrum is heavy and near-degenerate, $m_{\nu, 1}^{2} \simeq m_{\nu, 2}^{2} \simeq m_{\nu, 3}^{2}$, in which case we have

$$
\left[\begin{array}{l}
P_{e} \\
P_{\mu} \\
P_{\tau}
\end{array}\right]_{\text {near-degenerate }}=\left[\begin{array}{l}
0.3 \overline{3} \\
0.3 \overline{3} \\
0.3 \overline{3}
\end{array}\right] .
$$

\section{APPENDIX B: PROTON RECOILS AT BOREXINO}

To derive our bound from BOREXINO, we follow the method originally developed by $[85,86]$ for detecting muon and tau neutrinos from a supernova burst. The apparent electron equivalent recoil energy $E_{R}^{e}$ in a liquid scintillator is related to the proton recoil energy $E_{R}^{p}$ by Birks' formula for quenching,

$$
E_{R}^{e}\left(E_{R}^{p}\right)=\int_{0}^{E_{R}^{p}} d E_{R}^{\prime p}\left(1+k_{\mathrm{B}}\langle d T / d x\rangle\right)^{-1} .
$$

We only consider elastic scattering on hydrogen nuclei in the organic scintillator, as scattering on carbon is greatly quenched and unobservable [85,86]. Reference [87] estimated that the scattering rate per proton is restricted to be

$$
\begin{gathered}
R_{p}<2 \times 10^{-39} \mathrm{~s}^{-1} \quad \text { for } E_{R}^{e}>12.5 \mathrm{MeV} \\
\Rightarrow E_{R}^{p}>19.7 \mathrm{MeV} .
\end{gathered}
$$

The last line of this equation is obtained by numerically solving Eq. (B1) with a Birks' factor $k_{\mathrm{B}}=0.011 \mathrm{~cm} / \mathrm{MeV}$ [88] and by obtaining $\langle d T / d x\rangle$ (as a function of $E_{R}^{\prime p}$ ) with tables from [89] for toluene, a good approximation for the BOREXINO scintillator, pseudocumene.

The bound on $\left(G_{T}^{2} / G_{F}^{2}\right)\left\langle\sigma_{\mathrm{ann}} v\right\rangle$ is then obtained from Eqs. (1), (3) and (B2), where we now use the dipole form factor $F\left(q^{2}\right)=1 /\left(1+q^{2} /\left(0.71 \mathrm{GeV}^{2}\right)\right)^{2}$ [19] in Eq. (3). We note that the rate of inelastic processes that may induce fragmentation or excitation of the proton is highly suppressed for the momentum transfers considered here [19].
[1] J. F. Beacom, N. F. Bell, and G. D. Mack, Phys. Rev. Lett. 99, 231301 (2007).

[2] H. Yuksel, S. Horiuchi, J. F. Beacom, and S. Ando, Phys. Rev. D 76, 123506 (2007).

[3] E. Aprile et al. (XENON Collaboration), J. Cosmol. Astropart. Phys. 04 (2016) 027.

[4] D. S. Akerib et al. (LZ Collaboration), arXiv:1509.02910.

[5] J. Aalbers et al. (DARWIN Collaboration), J. Cosmol. Astropart. Phys. 11 (2016) 017.

[6] C. E. Aalseth et al., Eur. Phys. J. Plus 133, 131 (2018).

[7] https://indico.in2p3.fr/event/17777/, http://deap3600.ca/ deap-50t-detector/, https://goo.gl/re849A.

[8] J. Bramante, B. Broerman, R. F. Lang, and N. Raj, Phys. Rev. D 98, 083516 (2018).

[9] A. Olivares-Del Campo, C. Boehm, S. Palomares-Ruiz, and S. Pascoli, Phys. Rev. D 97, 075039 (2018).

[10] K. Abe et al. (XMASS Collaboration), Astropart. Phys. 89, 51 (2017).

[11] R. F. Lang, C. McCabe, S. Reichard, M. Selvi, and I. Tamborra, Phys. Rev. D 94, 103009 (2016).

[12] M. B. Voloshin, Phys. Rev. D 98, 034025 (2018).

[13] T. Kozynets, S. Fallows, and C. B. Krauss, Astropart. Phys. 105, 25 (2019).
[14] M. Schumann, L. Baudis, L. Bütikofer, A. Kish, and M. Selvi, J. Cosmol. Astropart. Phys. 10 (2015) 016.

[15] D. Franco et al., J. Cosmol. Astropart. Phys. 08 (2016) 017.

[16] D. G. Cerdeo, M. Fairbairn, T. Jubb, P. A. N. Machado, A. C. Vincent, and C. Bhm, J. High Energy Phys. 05 (2016) 118; 09 (2016) 48.

[17] J. L. Newstead, L. E. Strigari, and R. F. Lang, Phys. Rev. D 99, 043006 (2019).

[18] G. B. Gelmini, V. Takhistov, and S. J. Witte, arXiv:1812 .05550 .

[19] Y. Cui, M. Pospelov, and J. Pradler, Phys. Rev. D 97, 103004 (2018).

[20] J. F. Cherry, M. T. Frandsen, and I. M. Shoemaker, Phys. Rev. Lett. 114, 231303 (2015).

[21] B. Bertoni, S. Ipek, D. McKeen, and A. E. Nelson, J. High Energy Phys. 04 (2015) 170.

[22] B. Batell, T. Han, and B. Shams Es Haghi, Phys. Rev. D 97, 095020 (2018).

[23] B. Batell, T. Han, D. McKeen, and B. S. E. Haghi, Phys. Rev. D 97, 075016 (2018).

[24] G. Belanger and J. C. Park, J. Cosmol. Astropart. Phys. 03 (2012) 038.

[25] J. Huang and Y. Zhao, J. High Energy Phys. 02 (2014) 077. 
[26] K. Agashe, Y. Cui, L. Necib, and J. Thaler, J. Cosmol. Astropart. Phys. 10 (2014) 062.

[27] J. Berger, Y. Cui, and Y. Zhao, J. Cosmol. Astropart. Phys. 02 (2015) 005.

[28] K. Kong, G. Mohlabeng, and J. C. Park, Phys. Lett. B 743, 256 (2015).

[29] J. Kopp, J. Liu, and X. P. Wang, J. High Energy Phys. 04 (2015) 105.

[30] L. Necib, J. Moon, T. Wongjirad, and J. M. Conrad, Phys. Rev. D 95, 075018 (2017).

[31] H. Alhazmi, K. Kong, G. Mohlabeng, and J. C. Park, J. High Energy Phys. 04 (2017) 158.

[32] A. Bhattacharya, R. Gandhi, A. Gupta, and S. Mukhopadhyay, J. Cosmol. Astropart. Phys. 05 (2017) 002.

[33] D. Kim, J. C. Park, and S. Shin, Phys. Rev. Lett. 119, 161801 (2017).

[34] C. Kachulis et al. (Super-Kamiokande Collaboration), Phys. Rev. Lett. 120, 221301 (2018).

[35] G. F. Giudice, D. Kim, J. C. Park, and S. Shin, Phys. Lett. B 780, 543 (2018).

[36] A. Chatterjee, A. De Roeck, D. Kim, Z. G. Moghaddam, J. C. Park, S. Shin, L. H. Whitehead, and J. Yu, Phys. Rev. D 98, 075027 (2018).

[37] D. Kim, K. Kong, J. C. Park, and S. Shin, J. High Energy Phys. 08 (2018) 155.

[38] M. Aoki and T. Toma, J. Cosmol. Astropart. Phys. 10 (2018) 020 .

[39] C. Ha et al., Phys. Rev. Lett. 122, 131802 (2019).

[40] J. Berger, arXiv:1812.05616.

[41] R. Catena and P. Ullio, J. Cosmol. Astropart. Phys. 08 (2010) 004.

[42] M. Cirelli, G. Corcella, A. Hektor, G. Hütsi, M. Kadastik, P. Panci, M. Raidal, F. Sala, and A. Strumia, J. Cosmol. Astropart. Phys. 03 (2011) 051; 10 (2012) E01.

[43] F. Nesti and P. Salucci, J. Cosmol. Astropart. Phys. 07 (2013) 016.

[44] J. F. Navarro, C. S. Frenk, and S. D. M. White, Astrophys. J. 462, 563 (1996).

[45] G. Steigman, B. Dasgupta, and J. F. Beacom, Phys. Rev. D 86, 023506 (2012).

[46] A. Moline, J. A. Schewtschenko, S. Palomares-Ruiz, C. Bhm, and C. M. Baugh, J. Cosmol. Astropart. Phys. 08 (2016) 069.

[47] N. Klop and S. Ando, Phys. Rev. D 98, 103004 (2018).

[48] G. Bertone, A. R. Zentner, and J. Silk, Phys. Rev. D 72, 103517 (2005).

[49] G. Bertone, Phys. Rev. D 73, 103519 (2006).

[50] S. Horiuchi and S. Ando, Phys. Rev. D 74, 103504 (2006).

[51] P. Brun, G. Bertone, J. Lavalle, P. Salati, and R. Taillet, Phys. Rev. D 76, 083506 (2007).

[52] C. Arina, S. Kulkarni, and J. Silk, Phys. Rev. D 92, 083519 (2015).

[53] D. Z. Freedman, D. N. Schramm, and D. L. Tubbs, Annu. Rev. Nucl. Part. Sci. 27, 167 (1977).

[54] J. Erler and M. J. Ramsey-Musolf, Phys. Rev. D 72, 073003 (2005).

[55] M. Pospelov, Phys. Rev. D 84, 085008 (2011).

[56] R. H. Helm, Phys. Rev. 104, 1466 (1956).
[57] P. Giampa, Private communication.

[58] L. E. Strigari, New J. Phys. 11, 105011 (2009).

[59] J. Billard, L. Strigari, and E. Figueroa-Feliciano, Phys. Rev. D 89, 023524 (2014).

[60] E. Aprile et al. (XENON Collaboration), Phys. Rev. Lett. 121, 111302 (2018).

[61] G. Bellini et al. (Borexino Collaboration), Phys. Rev. C 81, 034317 (2010).

[62] A. Gando et al. (KamLAND Collaboration), Astrophys. J. 745, 193 (2012).

[63] R. Essig, M. Sholapurkar, and T. T. Yu, Phys. Rev. D 97, 095029 (2018).

[64] K. Bays et al. (Super-Kamiokande Collaboration), Phys. Rev. D 85, 052007 (2012).

[65] C. Garcia-Cely and J. Heeck, J. High Energy Phys. 05 (2017) 102.

[66] M. Pospelov, A. Ritz, and M. B. Voloshin, Phys. Lett. B 662, 53 (2008).

[67] L. G. van den Aarssen, T. Bringmann, and C. Pfrommer, Phys. Rev. Lett. 109, 231301 (2012).

[68] I. M. Shoemaker, Phys. Dark Universe 2, 157 (2013).

[69] P. Ko and Y. Tang, Phys. Lett. B 739, 62 (2014).

[70] M. Archidiacono, S. Hannestad, R. S. Hansen, and T. Tram, Phys. Rev. D 91, 065021 (2015).

[71] J. F. Cherry, A. Friedland, and I. M. Shoemaker, arXiv:1411 1071.

[72] A. Falkowski, J. Juknevich, and J. Shelton, arXiv:0908 .1790 .

[73] M. Pospelov and J. Pradler, Phys. Rev. D 89, 055012 (2014).

[74] M. Kaplinghat, L. Knox, and M. S. Turner, Phys. Rev. Lett. 85, 3335 (2000).

[75] V. D. Barger, W. Y. Keung, and G. Shaughnessy, Phys. Lett. B 664, 190 (2008).

[76] C. Rott, J. Siegal-Gaskins, and J. F. Beacom, Phys. Rev. D 88, 055005 (2013).

[77] N. Bernal, J. Martn-Albo, and S. Palomares-Ruiz, J. Cosmol. Astropart. Phys. 08 (2013) 011.

[78] C. Rott, S. In, J. Kumar, and D. Yaylali, J. Cosmol. Astropart. Phys. 11 (2015) 039.

[79] C. Rott, S. In, J. Kumar, and D. Yaylali, J. Cosmol. Astropart. Phys. 01 (2017) 016.

[80] N. F. Bell, S. Horiuchi, and I. M. Shoemaker, Phys. Rev. D 91, 023505 (2015).

[81] M. W. Goodman and E. Witten, Phys. Rev. D 31, 3059 (1985).

[82] A. Drukier and L. Stodolsky, Phys. Rev. D 30, 2295 (1984).

[83] I. Esteban, M. C. Gonzalez-Garcia, A. Hernandez-Cabezudo, M. Maltoni, and T. Schwetz, arXiv:1811.05487.

[84] http://www.nu-fit.org/.

[85] J. F. Beacom, W. M. Farr, and P. Vogel, Phys. Rev. D 66, 033001 (2002).

[86] B. Dasgupta and J. F. Beacom, Phys. Rev. D 83, 113006 (2011).

[87] T. Bringmann and M. Pospelov, arXiv:1810.10543.

[88] G. Bellini et al. (Borexino Collaboration), Phys. Rev. D 89, 112007 (2014).

[89] https://physics.nist.gov/PhysRefData/Star/Text/PSTAR.html. 